\title{
Moments characterization of order 3 matrix exponential distributions ${ }^{\star}$
}

\author{
András Horváth ${ }^{1}$, Sándor Rácz ${ }^{2}$, and Miklós Telek ${ }^{2}$ \\ 1 Dipartimento di Informatica, University of Torino, Italy \\ 2 Technical University of Budapest, Hungary \\ horvath@di.unito.it, sandor.racz.74@gmail.com, telek@hit. bme.hu
}

\begin{abstract}
The class of order 3 phase type distributions $(\mathrm{PH}(3))$ is known to be a proper subset of the class of order 3 matrix exponential distributions $(\mathrm{ME}(3))$. In this paper we investigate the relation of these two sets for what concerns their moment bounds.

To this end we developed a procedure to check if a matrix exponential function of order 3 defines a $\mathrm{ME}(3)$ distribution or not. This procedure is based on the time domain analysis of the density function. The proposed procedure requires the numerical solution of a transcendent equation in some cases.

The presented moment bounds are based on some unproved conjectures which are verified only by numerical investigations.

Keywords: Matrix exponential distributions, Phase type distributions, moment bounds.
\end{abstract}

\section{Introduction}

The availability of efficient matrix analytic methods (see e.g., $[7,10]$ ) reinforced the research of distributions with matrix exponential representation. The order of these distributions is defined as the (minimal) cardinality of the matrix that describes the distribution. The two main classes of these distributions are the class of phase type distributions $[8,9]$, which has a nice stochastic interpretation due to its underlying continuous time Markov chain, and the class of matrix exponential distributions [1], which does not allow for a simple stochastic interpretation.

It has been known for a long time that considering distributions of order 2 the two classes are identical, $\operatorname{ME}(2) \equiv \mathrm{PH}(2)$, but for $n>2 \mathrm{PH}(\mathrm{n})$ is a proper subset of $\mathrm{ME}(\mathrm{n})$ [12]. Unfortunately there are no tools to investigate the relation of the $\mathrm{ME}(\mathrm{n})$ and the $\mathrm{PH}(\mathrm{n})$ classes for $n>2$. However, recent results on $\mathrm{ME}(3)$ [5] and $\mathrm{PH}(3)$ [6] distributions make it possible to investigate the relation of the $\mathrm{ME}(3)$ and the $\mathrm{PH}(3)$ classes.

The practical importance of low order $\mathrm{PH}$ and $\mathrm{ME}$ distributions comes from the fact that the complexity of the matrix analytic analysis increases rapidly

\footnotetext{
* This work is partially supported by the NAPA-WINE FP7 project and by the OTKA K61709 grant.
} 
with the order of the model components (e.g., PH distribution of the service time). Recent results suggest that matrix analytic methods are applicable for models with matrix exponential distributions as well as for models with phase type distributions [2]. Consequently, one can gain if the durations to be modelled can be described by a ME distribution with lower order than the application of a $\mathrm{PH}$ distributions would require.

We compare the flexibility of the $\mathrm{ME}(3)$ and the $\mathrm{PH}(3)$ classes through their moment bounds. It is not the only and not necessarily the easiest way to compare them, but this choice is motivated by the fact that moments and related measures (e.g., coefficient of variation) are the most frequently used parameters of distributions.

This paper is strongly related to the extensive work of Mark Fackrell in [5]. We reconsider some questions of [5] and complement those results with alternative ones. The main goal of this paper is to answer the following question ([5] p. 110) "The class of $\mathrm{PH}$ distributions is a proper subset of the class of ME distributions, but how much larger is the latter class than the former?". In [5] the question is answered for $\mathrm{ME}(\mathrm{n})$ and $\cup_{m \geq n} \mathrm{PH}(\mathrm{m})$. We believe that this question has more practical importance for $\mathrm{ME}(\mathrm{n})$ and $\mathrm{PH}(\mathrm{n})$. In this work we try to answer this question for $\mathrm{ME}(3)$ and $\mathrm{PH}(3)$.

Related ME(3) results: [5] devotes its main attention to the matrix exponential distributions of order $n>2$ and provides important necessary conditions for being a member of $\mathrm{ME}(\mathrm{n})$. Additionally, [5] provides necessary and sufficient conditions for being a member of $\mathrm{ME}(3)$. These conditions are given in the Laplace transform domain. Assuming that for a given triple $\left\{b_{1}, b_{2}, b_{3}\right\}$ the Laplace transform of a matrix exponential function takes the form

$$
\frac{x_{2} s^{2}+x_{1} s+b_{1}}{s^{3}+b_{3} s^{2}+b_{2} s+b_{1}}
$$

(i.e., there is no probability mass at 0 ) the linear and parametric curves provided in [5] bound the region of $\left\{x_{1}, x_{2}\right\}$ where the matrix exponential function is a member of the $\mathrm{ME}(3)$ class.

Unfortunately, we did not find an easy implementation of these transform domain constraints, and this is why we developed a time domain counterpart for $\mathrm{ME}(3)$ characterization.

An important property of the $\mathrm{ME}(3)$ class, namely its minimal coefficient of variation, is studied in [4]. The results provided here verify the ones provided there.

Related PH(3) results: Another important preliminary work is [6] which provides a canonical representation of $\mathrm{PH}(3)$ distributions. More precisely, [6] presents an algorithm that transforms any order 3 matrix exponential function to $\mathrm{PH}(3)$ canonical form if it is possible. In this paper, this algorithm is used to characterize the borders of the $\mathrm{PH}(3)$ class.

The rest of the paper is organized as follows. Section 2 defines the class of matrix exponential distributions and the basic notations. Section 3 presents a procedure to check if a matrix exponential function of order 3 is a member of 
the $\mathrm{ME}(3)$ class or not. Using this procedure and its counterpart for the $\mathrm{PH}(3)$ class from [6], Section 4 investigates the relation of the moment bounds of these two classes. The paper is concluded in Section 5 .

\section{Matrix exponential distributions}

Definition 1. The vector matrix pair $(\underline{v}, \boldsymbol{H})$ defines a matrix exponential distribution iff

$$
F(t)=\operatorname{Pr}(X<t)=1-\underline{v} e^{\boldsymbol{H}_{t}} \mathbb{I}, \quad t \geq 0
$$

is a valid cumulative distribution function, i.e., $F(0) \geq 0, \lim _{t \rightarrow \infty} F(t)=1$ and $F(t)$ is monotone increasing.

In (1), the row vector, $\underline{v}$, is referred to as the initial vector, the square matrix, $\boldsymbol{H}$, as the generator and $\mathbb{I}$ as the closing vector. Without loss of generality (see [8]), throughout this paper we assume that the closing vector is a column vector of ones, i.e., $\mathbb{I}=[1,1, \ldots, 1]^{T}$.

The density, the Laplace transform and the moments of the matrix exponential distribution defined by $(\underline{v}, \boldsymbol{H})$ are

$$
\begin{gathered}
f(t)=\underline{v} e^{\boldsymbol{H}_{t}}(-\boldsymbol{H}) \mathbb{I}, \\
f^{*}(s)=E\left(e^{-s X}\right)=\underline{v}(s \boldsymbol{I}-\boldsymbol{H})^{-1}(-\boldsymbol{H}) \mathbb{I}, \\
\mu_{n}=E\left(X^{n}\right)=n ! \underline{v}(-\boldsymbol{H})^{-n} \mathbb{I} .
\end{gathered}
$$

To ensure that $\lim _{t \rightarrow \infty} F(t)=1, \boldsymbol{H}$ has to fulfill the necessary condition that the real parts of its eigenvalues are negative (consequently $\boldsymbol{H}$ is non-singular).

The remaining constraint is the monotonicity of $F(t)$. It is the most difficult property to check. Instead of checking if $F(t)$ is monotone increasing, in the next section, we check if $f(t)$ is non-negative.

\section{$3 \quad$ Matrix exponential distributions of order 3}

We subdivide the class of $\mathrm{ME}(3)$ distributions according to the eigenvalue structure of $\boldsymbol{H}$. With $\lambda_{1}, \lambda_{2}, \lambda_{3}$ denoting the eigenvalues of the matrix $-\boldsymbol{H}$, we have the following possible cases:

- class A: $\lambda_{1}, \lambda_{2}, \lambda_{3} \in \mathbb{R}^{+}, \lambda_{1}<\lambda_{2}<\lambda_{3}$

- class B: $\lambda_{1}, \lambda_{2}, \lambda_{3} \in \mathbb{R}^{+}, \lambda_{1}=\lambda_{2}<\lambda_{3}$ or $\lambda_{1}<\lambda_{2}=\lambda_{3}$

- class C: $\lambda_{1}=\lambda_{2}=\lambda_{3} \in \mathbb{R}^{+}$,

- class D: $\lambda_{1} \in \mathbb{R}^{+}, \lambda_{2}=\bar{\lambda}_{3} \in \mathbb{C}^{+}$,

where $\mathbb{R}^{+}$denotes the set of strictly positive real numbers and $\mathbb{C}^{+}$the set of complex numbers with strictly positive real part. The following subsections consider these four cases. 


\subsection{Case A: 3 different real eigenvalues}

In this case the general form of the density function and its derivative are

$$
\begin{aligned}
f(t) & =a_{1} e^{-\lambda_{1} t}+a_{2} e^{-\lambda_{2} t}+a_{3} e^{-\lambda_{3} t} \\
f^{\prime}(t) & =-a_{1} \lambda_{1} e^{-\lambda_{1} t}-a_{2} \lambda_{2} e^{-\lambda_{2} t}-a_{3} \lambda_{3} e^{-\lambda_{3} t}
\end{aligned}
$$

Without loss of generality, we check the non-negativity of $f(t)$ assuming that $\lambda_{1}<\lambda_{2}<\lambda_{3}$.

Theorem 1. $f(t)$ is non-negative for $t \geq 0$ iff

$$
\begin{aligned}
& -a_{1}+a_{2}+a_{3} \geq 0 \text { and } \\
& -a_{1}>0 \text { and } \\
& - \text { if } a_{2}<-a_{1} \frac{\lambda_{3}-\lambda_{1}}{\lambda_{3}-\lambda_{2}} \text { then } a_{3} \geq a_{1} \frac{\lambda_{2}-\lambda_{1}}{\lambda_{3}-\lambda_{2}}\left(-\frac{a_{2}}{a_{1}} \frac{\lambda_{3}-\lambda_{2}}{\lambda_{3}-\lambda_{1}}\right)^{\frac{\lambda_{3}-\lambda_{1}}{\lambda_{2}-\lambda_{1}}} .
\end{aligned}
$$

Proof. First, we note that $f(t)$ is a monotone increasing function of $a_{1}, a_{2}$ and $a_{3}$ for $t \geq 0$ and both $f(t)$ and $f^{\prime}(t)$ can have at most 2 roots in $(0, \infty)$ (excluding 0 and infinity).

The non-negativity of $f(t)$ at $t=0$ results in the first condition and the non-negativity of $f(t)$ at $t \rightarrow \infty$ results in the second condition of the theorem.

In the rest we suppose that $a_{1}>0$ and $a_{1}+a_{2}+a_{3} \geq 0$. We investigate the non-negativity of $f(t)$ by constructing $f^{*}(t)=a_{1} e^{-\lambda_{1} t}+a_{2} e^{-\lambda_{2} t}+a_{3}^{*} e^{-\lambda_{3} t}$ such that $a_{3}^{*}$ takes the minimal $a_{3}$ value with which $f(t)$ is still non-negative, i.e., we will have $f^{*}(c)=0$ for some $c \geq 0$.

We have the following two cases:

a) $f^{*}(c)$ touches the x-axes at $c>0$, that is, $f^{*}(c)=0$ and $f^{\prime *}(c)=0$,

b) $f^{*}(0)=0$ and $f^{*}(0) \geq 0$.

In case a) we have

$$
\begin{aligned}
f^{*}(c) & =a_{1} e^{-\lambda_{1} c}+a_{2} e^{-\lambda_{2} c}+a_{3}^{*} e^{-\lambda_{3} c}=0, \\
f^{\prime *}(c) & =-a_{1} \lambda_{1} e^{-\lambda_{1} c}-a_{2} \lambda_{2} e^{-\lambda_{2} c}-a_{3}^{*} \lambda_{3} e^{-\lambda_{3} c}=0,
\end{aligned}
$$

from which

$$
\begin{aligned}
& \frac{a_{2}}{a_{1}}=-\frac{\lambda_{3}-\lambda_{1}}{\lambda_{3}-\lambda_{2}} e^{\left(\lambda_{2}-\lambda_{1}\right) c}, \\
& \frac{a_{3}^{*}}{a_{1}}=\frac{\lambda_{2}-\lambda_{1}}{\lambda_{3}-\lambda_{2}} e^{\left(\lambda_{3}-\lambda_{1}\right) c} .
\end{aligned}
$$

If $a_{2} \geq-a_{1} \frac{\lambda_{3}-\lambda_{1}}{\lambda_{3}-\lambda_{2}}$ then there is no $c>0$ that satisfies (9), since the left hand side of (9) is negative and less than $-\frac{\lambda_{3}-\lambda_{1}}{\lambda_{3}-\lambda_{2}}$. Consequently, case a) is not possible when $a_{2} \geq-a_{1} \frac{\lambda_{3}-\lambda_{1}}{\lambda_{3}-\lambda_{2}}$. 
If $a_{2}<-a_{1} \frac{\lambda_{3}-\lambda_{1}}{\lambda_{3}-\lambda_{2}}$ then $c$ is obtained from (9) as

$$
c=\frac{\log \left(-\frac{a_{2}}{a_{1}} \frac{\lambda_{3}-\lambda_{2}}{\lambda_{3}-\lambda_{1}}\right)}{\lambda_{2}-\lambda_{1}},
$$

and substituting it to (10) gives

$$
a_{3}^{*}=a_{1} \frac{\lambda_{2}-\lambda_{1}}{\lambda_{3}-\lambda_{2}}\left(-\frac{a_{2}}{a_{1}} \frac{\lambda_{3}-\lambda_{2}}{\lambda_{3}-\lambda_{1}}\right)^{\frac{\lambda_{3}-\lambda_{1}}{\lambda_{2}-\lambda_{1}}} .
$$

In case b) we have

$$
\begin{aligned}
f^{*}(0) & =a_{1}+a_{2}+a_{3}^{*}=0, \\
f^{\prime *}(0) & =-a_{1} \lambda_{1}-a_{2} \lambda_{2}-a_{3}^{*} \lambda_{3} \geq 0 .
\end{aligned}
$$

Substituting $a_{3}^{*}=-a_{1}-a_{2}$ from (11) into (12) we have that (12) holds when $a_{2} \geq-a_{1} \frac{\lambda_{3}-\lambda_{1}}{\lambda_{3}-\lambda_{2}}$.

\subsection{Case B: 2 different real eigenvalues}

In this case we have two options.

- The multiplicity of the dominant eigenvalue, $\lambda_{1},\left(\lambda_{1}<\lambda_{2}\right)$ is one and hence the general form of the density function is

$$
f_{1}(t)=a_{1} e^{-\lambda_{1} t}+\left(a_{2}+a_{21} t\right) e^{-\lambda_{2} t} .
$$

- The multiplicity of the dominant eigenvalue, $\lambda_{1},\left(\lambda_{1}<\lambda_{2}\right)$ is two and hence the general form of the density function is

$$
f_{2}(t)=\left(a_{1}+a_{11} t\right) e^{-\lambda_{1} t}+a_{2} e^{-\lambda_{2} t} .
$$

Theorem 2. $f_{1}(t)$ is non-negative for $t \geq 0$ iff

$$
\begin{aligned}
& -a_{1}+a_{2}>0 \text { and } \\
& -a_{1} \geq 0 \text { and } \\
& -a_{21} \geq a_{21}^{*}
\end{aligned}
$$

where $a_{21}^{*}$ is that solution of

$$
a_{21} e^{a_{2} \lambda_{2} / a_{21}}+a_{1}\left(\lambda_{2}-\lambda_{1}\right) e^{1+a_{2} \lambda_{1} / a_{21}}=0
$$

which satisfies $a_{21}\left(1-\left(\lambda_{2}-\lambda_{1}\right)\right)>a_{2}\left(\lambda_{2}-\lambda_{1}\right)$. 
Proof. $f_{1}(t)$ is a monotone increasing function of $a_{1}, a_{2}$ and $a_{21}$ for $t \geq 0$ and both $f_{1}(t)$ and $f_{1}^{\prime}(t)$ can have at most 2 roots in $(0, \infty)$ (excluding 0 and infinity).

The non-negativity of $f_{1}(t)$ at $t=0$ results in the first condition and the non-negativity of $f_{1}(t)$ at $t \rightarrow \infty$ results in the second condition. The minimal $a_{21}$ value for which $f_{1}(t)$ is non-negative is obtained assuming that $f_{1}(t)$ touches the $\mathrm{x}$ axes at $t=c>0$, i.e., $f_{1}(c)=0$ and $f_{1}^{\prime}(c)=0$. Solving this set of equations for $a_{21}$ and $c$, we have

$$
c=\frac{a_{21}-a_{2}\left(\lambda_{2}-\lambda_{1}\right)}{a_{21}\left(\lambda_{2}-\lambda_{1}\right)},
$$

and (15) and the $a_{21}\left(1-\left(\lambda_{2}-\lambda_{1}\right)\right)>a_{2}\left(\lambda_{2}-\lambda_{1}\right)$ condition is equivalent with $c>0$.

Theorem 3. $f_{2}(t)$ is non-negative for $t \geq 0$ iff

$$
\begin{aligned}
& -a_{1}+a_{2}>0 \text { and } \\
& -a_{11} \geq 0 \text { and } \\
& -a_{11} \geq a_{11}^{*}
\end{aligned}
$$

where $a_{11}^{*}$ is that solution of

$$
a_{2} e^{\lambda_{2}\left(\frac{a_{1}}{a_{11}}-\frac{1}{\lambda_{2}-\lambda_{1}}\right)}-a_{11}\left(\lambda_{2}-\lambda_{1}\right) e^{\lambda_{1}\left(\frac{a_{1}}{a_{11}}-\frac{1}{\lambda_{2}-\lambda_{1}}\right)}=0
$$

which satisfies $-a_{1}\left(\lambda_{2}-\lambda_{1}\right)>a_{11}\left(1+\lambda_{2}-\lambda_{1}\right)$.

Proof. The proof follows the same pattern as the one for $f_{1}(t)$.

It has to be noted that the third condition of Theorem 2 and 3 are transcendent, and consequently, numerical methods are required to compute them.

\subsection{Case C: 1 real eigenvalue}

In this case the general form of the density function is

$$
f(t)=\left(a_{0}+a_{1} t+a_{2} t^{2}\right) e^{-\lambda t}
$$

Theorem 4. $f(t)$ is non-negative for $t \geq 0$ iff

$-a_{0}>0$ and

$-a_{2}>0$ and

$-a_{1} \geq-2 \sqrt{a_{0} a_{2}}$.

Proof. $f(t)$ is a monotone increasing function of $a_{0}, a_{1}$ and $a_{2}$ for $t \geq 0$ and both $f(t)$ and $f^{\prime}(t)$ can have at most 2 roots in $(0, \infty)$ (excluding 0 and infinity).

The non-negativity of $f(t)$ at $t=0$ results in the first condition and the non-negativity of $f(t)$ at $t \rightarrow \infty$ results in the second condition.

Supposing that $a_{0}>0$ and $a_{2}>0$ we have the following two cases:

- if $a_{1} \geq 0$ then $a_{0}+a_{1} t+a_{2} t^{2}$ is monotone increasing on $(0, \infty)$,

- if $a_{1}<0$ then $a_{0}+a_{1} t+a_{2} t^{2}$ has a minimum at $t=-\frac{a_{1}}{2 a_{2}}$ which is $a_{0}-\frac{a_{1}^{2}}{4 a_{2}}$.

From which the third condition comes. 


\subsection{Case D: one real and a pair of complex eigenvalues}

In this case the general form of the density function is

$$
f(t)=a_{1} e^{-\lambda_{1} t}+a_{2} \cos (\omega t+\phi) e^{-\lambda_{c} t}
$$

where, for uniqueness, $a_{2}$ and $\phi$ are defined such that $a_{2}>0$ and $-\pi<\phi \leq \pi$.

Theorem 5. $f(t)$ is non-negative for $t \geq 0$ iff

$$
\begin{aligned}
& -a_{1}+a_{2} \cos (\phi)>0 \text { and } \\
& -a_{1}>0 \text { and } \\
& -\lambda_{1} \leq \lambda_{c} \text { and } \\
& -a_{2}<a_{1} e^{\left(\lambda_{c}-\lambda_{1}\right) \frac{2 \pi}{\omega}} \text { and } \\
& - \text { if } a_{1}<a_{2}\left(<a_{1} e^{\left(\lambda_{c}-\lambda_{1}\right) \frac{2 \pi}{\omega}}\right) \text { then } f(\check{t}) \geq 0 \text { and } f(\hat{t}) \geq 0 \text { and } \\
& - \text { if } a_{1}<a_{2}\left(<a_{1} e^{\left(\lambda_{c}-\lambda_{1}\right) \frac{2 \pi}{\omega}}\right) \text { and } f^{\prime}(t) \text { has roots in }[\check{t}, \hat{t}] \text { then } f(t) \geq 0 \text { at those } \\
& \quad \text { roots, }
\end{aligned}
$$

where $\check{t}=\max \left(0, \frac{\pi-2 \phi}{2 \omega}\right)$ and $\hat{t}=\min \left(\frac{1}{\lambda_{c}-\lambda_{1}} \log \left(\frac{a_{2}}{a_{1}}\right), \frac{\pi-\phi}{\omega}\right)$.

Proof. The non-negativity of $f(t)$ at $t=0$ results in the first condition and the non-negativity of $f(t)$ at $t \rightarrow \infty$ results in the second and the third conditions. The sign of $f(t)$ is determined by two main factors:

- the relation of the two exponential functions $a_{1} e^{-\lambda_{1} t}$ and $a_{2} e^{-\lambda_{c} t}$,

- the value of the cyclic term $\cos (\omega t+\phi)$.

Supposing that the first 3 conditions hold, $f(t)$ has the following properties:

- $f(t)$ is a monotone increasing function of $a_{1}$ for $t \geq 0$.

- Both $f(t)$ and $f^{\prime}(t)$ might have infinitely many roots in $(0, \infty)$ (excluding 0 and infinity).

- If $a_{1}>a_{2}$ then $a_{1} e^{-\lambda_{1} t}>a_{2} e^{-\lambda_{c} t}$ for $\forall t>0$, and consequently $f(t)>0$ for $\forall t>0$. If $a_{1}<a_{2}$ and $\lambda_{1}<\lambda_{c}$ then $a_{1} e^{-\lambda_{1} t}>a_{2} e^{-\lambda_{c} t}$ for $\forall t>t_{r}=$ $\frac{1}{\lambda_{c}-\lambda_{1}} \log \left(\frac{a_{2}}{a_{1}}\right)$, and consequently $f(t)>0$ for $\forall t>t_{r}$, since $t_{r}$ is the solution of $a_{1} e^{-\lambda_{1} t_{r}}>a_{2} e^{-\lambda_{c} t_{r}}$ and the quicker decay of $e^{-\lambda_{c} t}$ ensures the last statement.

- If at the end of the first period of $\cos (\omega t+\phi)$, i.e., at $t_{p}=\frac{2 \pi}{\omega}$ we have $a_{1} e^{-\lambda_{1} t_{p}}<a_{2} e^{-\lambda_{c} t_{p}}$, then for $t=\frac{\pi-\phi}{\omega}<t_{p}$ we have

$$
\begin{aligned}
f\left(\frac{\pi-\phi}{\omega}\right) & =a_{1} e^{-\lambda_{1} \frac{\pi-\phi}{\omega}}+a_{2} \cos \left(\omega \frac{\pi-\phi}{\omega}+\phi\right) e^{-\lambda_{c} \frac{\pi-\phi}{\omega}} \\
& =a_{1} e^{-\lambda_{1} \frac{\pi-\phi}{\omega}}-a_{2} e^{-\lambda_{c} \frac{\pi-\phi}{\omega}}<a_{1} e^{-\lambda_{1} t_{p}}-a_{2} e^{-\lambda_{c} t_{p}}<0 .
\end{aligned}
$$

- If $f(t)>0$ for $\forall t>0$ for a given $a_{2}$ then $\tilde{f}(t)=a_{1} e^{-\lambda_{1} t}+\tilde{a}_{2} \cos (\omega t+$ b) $e^{-\lambda_{c} t}>0$ for $\forall t>0$ and $\forall \tilde{a}_{2} \in\left[0, a_{2}\right]$.

- If $f(t) \geq 0$ for $\forall t \in\left[0, t_{p}\right]$ then $f(t) \geq 0$ for $\forall t>0$, because the non-negativity of $f(t)$ for $\left[t_{p}, \infty\right)$ is equivalent with the non-negativity of $a_{1} e^{-\lambda_{1} t}+\tilde{a}_{2} \cos (\omega t+\phi) e^{-\lambda_{c} t}$, where $\tilde{a}_{2}=a_{2} e^{-\left(\lambda_{c}-\lambda_{1}\right) t_{p}} \leq a_{2}$. 
According to these properties, if $\lambda_{1}<\lambda_{c}$,

$-a_{2} \leq a_{1}$ implies that $f(t)$ is non-negative.

$-a_{2}>a_{1} e^{\left(\lambda_{c}-\lambda_{1}\right) \frac{2 \pi}{\omega}}$ or equivalently $t_{r}>t_{p}$ implies that $f(t)$ is not nonnegative,

- if $a_{1}<a_{2} \leq a_{1} e^{\left(\lambda_{c}-\lambda_{1}\right) \frac{2 \pi}{\omega}}$ then $f(t)$ can become negative depending on the initial phase of the cosine term. $f(t)$ can become negative only when the cosine term is negative, e.g., $t \in\left(\frac{\pi-2 \phi}{2 \omega}, \frac{3 \pi-2 \phi}{2 \omega}\right)$, but due to the faster decay of the $e^{-\lambda_{c} t}$ term it is enough to study the first interval, where the cosine term takes the values from 0 to -1 , i.e., $\left(\frac{\pi-2 \phi}{2 \omega}, \frac{\pi-\phi}{\omega}\right)$. Depending on the initial phase, $\phi, \frac{\pi-2 \phi}{2 \omega}$ can be less than 0 and $\frac{\pi-\phi}{\omega}$ can be greater than $t_{r}$. Considering these additional constraints $\check{t}$ and $\hat{t}$ defines the borders of the decisive interval. If $f(t)$ is non-negative on $[\check{t}, \hat{t}]$ it is non-negative for $\forall t>0$. $f^{\prime}(\check{t})<0$ because both $e^{-\lambda_{1} t}$ and $\cos (\omega t+\phi) e^{-\lambda_{c} t}$ decay at $t=\check{t}$.

If $f^{\prime}(\hat{t})$ is non-positive, $f^{\prime}(t)$ has 0,1 , or 2 roots in $[\check{t}, \hat{t}]$, and the sign of $f(t)$ at these roots decides the non-negativity of $f(t)$.

If $f^{\prime}(\hat{t})$ is positive, $f(t)$ has a single minimum in $[\check{t}, \hat{t}]$, and the sign of this minimum decides the non-negativity of $f(t)$.

\section{Moments bounds of the $\mathrm{ME}(3)$ class}

The previous section provides results to check the ME(3) membership of order 3 matrix exponential functions. We implemented those checks in a Mathematica function. Using this implementation, in this section, we numerically investigate the flexibility of the $\mathrm{ME}(3)$ class compared to the limits of the $\mathrm{PH}(3)$ class, for which similar results are provided in [6] to check $\mathrm{PH}(3)$ membership.

A continuous $\mathrm{ME}(3)$ or $\mathrm{PH}(3)$ distribution is uniquely characterized by its first 5 moments. For a given set of $\left\{\mu_{1}, \ldots, \mu_{5}\right\}$ moments we check the $\operatorname{ME}(3)$ and $\mathrm{PH}(3)$ membership with a two step procedure.

- The first step is to compute a vector and matrix pair of order $3,(\underline{v}, \boldsymbol{H})$, for which $i ! \underline{v}(-\boldsymbol{H})^{-i} \mathbb{I}=\mu_{i}, i=1, \ldots, 5$. The procedure of Appie van de Liefvoort in [12] provides such $(\underline{v}, \boldsymbol{H})$ pair with a proper transformation of the closing vector ${ }^{3}$.

- Starting from $(\underline{v}, \boldsymbol{H})$, if the $\mathrm{PH}(3)$ transformation procedure in [6] generates a valid canonical representation then $\left\{\mu_{1}, \ldots \mu_{5}\right\}$ represents a member of the $\mathrm{PH}(3)$ set. Similarly, if the matrix exponential function, $\underline{v} e^{\boldsymbol{H}_{t}}(-\boldsymbol{H}) \mathbb{I}$, is non-negative according to the checks of the previous section then $\left\{\mu_{1}, \ldots \mu_{5}\right\}$ represents a member of the $\operatorname{ME}(3)$ set.

As in previous works, to reduce the number of parameters we introduce the normalized moments, $n_{i}=\frac{\mu_{i}}{\mu_{i-1} \mu_{1}}$, which eliminate a scaling factor and represent the shape of $\mathrm{ME}(3)$ and $\mathrm{PH}(3)$ distributions with 4 parameters, $\left\{n_{2}, n_{3}, n_{4}, n_{5}\right\}$.

\footnotetext{
${ }^{3}$ In $[12]$ the initial and the closing vectors are $\{1,0,0, \ldots, 0\}$. In our case the closing vector is $\{1,1, \ldots, 1\}$, hence a similarity transformation is required as described in [11].
} 
The subsequent numerical results are divided into investigations of the $n_{2}, n_{3}$ domain with arbitrary $n_{4}, n_{5}$ and investigations of the $n_{4}, n_{5}$ domain with given $n_{2}, n_{3}$.

\subsection{The second and third normalized moments}

The $n_{2}, n_{3}$ normalized moment bounds of the $\mathrm{PH}(3)$ class are not completely known yet. There is a proved result for the valid range of the $\mathrm{APH}(3)$ class [3], and there is a numerically checked conjecture that the related borders of the $\mathrm{PH}(3)$ class coincide with the ones of the $\mathrm{APH}(3)$ class [6]. Here we compare the borders of the $\mathrm{ME}(3)$ class with these borders of the $\mathrm{PH}(3)$ class.

To check if an $n_{2}, n_{3}$ pair is inside the range of the $\mathrm{ME}(3)$ class is rather difficult. We have tools to check if $\left\{n_{2}, n_{3}, n_{4}, n_{5}\right\}$ defines an ME(3) distribution. Based on this tool, for a given $n_{2}, n_{3}$ pair a natural procedure would be to check the $\mathrm{ME}(3)$ membership of $\left\{n_{2}, n_{3}, x, y\right\}$, where $x$ and $y$ run through the positive quarter plain. Unfortunately, this procedure is infeasible, because it is practically impossible to find valid $n_{4}, n_{5}$ pairs with exhaustive search.

To get around this problem we applied special ME(3) subclasses whose structure is defined by 2 shape parameters and a scaling factor. Having these subclasses we set the 2 shape parameters to match $n_{2}, n_{3}$ and checked if we obtained a valid distribution.

The Exp-Erlang and the Erlang-Exp distributions in [3] form such subsets, which we used for $n_{2}, n_{3}$ pairs inside the range of the $\mathrm{PH}(3)$ class.

For $n_{2}, n_{3}$ pairs outside the range of the $\mathrm{PH}(3)$ class we used the following function with complex roots $\left(a_{1}=a_{2}=a, \lambda_{1}=\lambda_{2}=\lambda\right.$ in (19))

$$
f(t)=a e^{-\lambda t}(1+\cos (\omega t+\phi))
$$

where $a$ is a normalizing constant $\left(\int_{t} e^{-\lambda t}(1+\cos (\omega t+\phi)) d t=1 / a\right), \lambda$ is the scaling factor and $\omega$ and $\phi$ are the two shape parameters. When $\lambda=1$

$$
\begin{aligned}
& n_{2}=\frac{2\left(\sqrt{1+\omega^{2}}+\cos (\phi+\arctan (\omega))\right)\left(\left(1+\omega^{2}\right)^{\frac{3}{2}}+\cos (\phi+3 \arctan (\omega))\right)}{\left(1+\omega^{2}+\cos (\phi+2 \arctan (\omega))\right)^{2}}, \\
& n_{3}=\frac{3\left(\sqrt{1+\omega^{2}}+\cos (\phi+\arctan (\omega))\right)\left(\left(1+\omega^{2}\right)^{2}+\cos (\phi+4 \arctan (\omega))\right)}{\left(1+\omega^{2}+\cos (\phi+2 \arctan (\omega))\right)\left(\left(1+\omega^{2}\right)^{\frac{3}{2}}+\cos (\phi+3 \arctan (\omega))\right)} .
\end{aligned}
$$

For a given $n_{2}, n_{3}$ pair solving this equation for the $\phi, \omega$ pair gives a matrix exponential function whose second and third normalized moments are $n_{2}$ and $n_{3}$. The non-negativity of this function can be checked by Theorem 5 .

Figure 1 depicts the borders of the $\mathrm{ME}(3)$ class (obtained for subclass (20)) and the borders of the $\mathrm{PH}(3)$ class (inner borders of the figure) on the $n_{2}, n_{3}$ plain. Our numerical investigations suggest that the outer borders in Figure 1 are the borders of the whole $\mathrm{ME}(3)$ class, but we cannot prove it. The left most point of these borders, $n_{2}=1.200902$ gives the $\mathrm{ME}(3)$ distribution with 
minimal $n_{2}$ or, equivalently, with minimal coefficient of variation, and this point corresponds to the minimal coefficient of variation of the $\mathrm{ME}(3)$ class reported in [4]. The $\mathrm{PH}(3)$ class, and consequently the $\mathrm{ME}(3)$ class, are known to be only lower bounded when $n_{2}>1.5$. That is why the upper bound curves end at $n_{2}=1.5$.

The results of Section 3 indicate already that the borders of the ME(3) class do not exhibit nice closed form expressions, but numerical methods are required for their evaluation. We used the standard floating point precision of Mathematica to compute the presented results, but these computations are numerically sensitive.

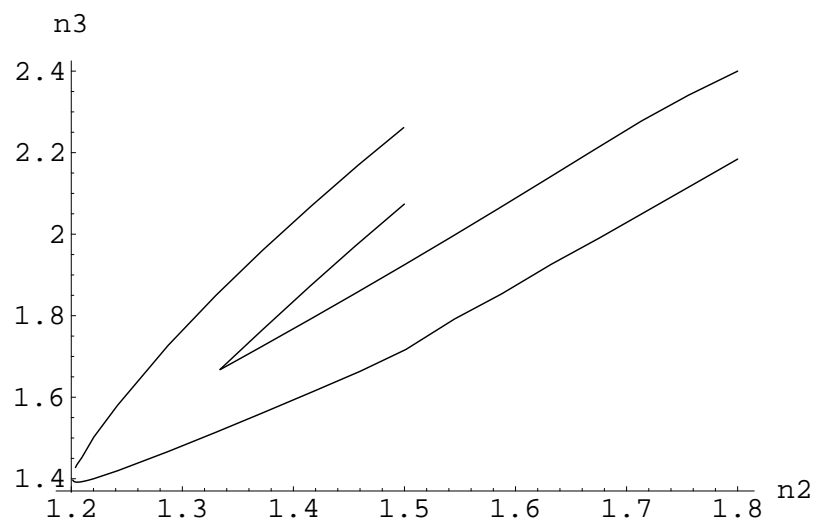

Fig. 1. The range of second and third normalized moments of the $\mathrm{PH}(3)$ and $\mathrm{ME}(3)$ classes

\subsection{The fourth and fifth normalized moments}

In this section we study the region of realizable fourth and fifth normalized moments $\left(n_{4}\right.$ and $\left.n_{5}\right)$ for a given pair of second and third normalized moments $\left(n_{2}\right.$ and $\left.n_{3}\right)$. In order to find this region we make use of the subclasses presented in Section 4.1. We use Erlang-Exp distributions [3] inside the $\mathrm{PH}(3)$ borders of Figure 1 and the subclass defined by (20) between the $\mathrm{PH}(3)$ and the $\mathrm{ME}(3)$ borders. First we generate a matrix exponential function from the given $\mathrm{ME}(3)$ subclass that realizes the pair $\left(n_{2}, n_{3}\right)$. Then we calculate $n_{4}$ and $n_{5}$ for this matrix exponential function and use them as starting point in exploring the realizable region of $n_{4}$ and $n_{5}$. Since the realizable region of the $\mathrm{PH}(3)$ class is a subregion of the realizable region of the $\mathrm{ME}(3)$ class, it is easier to start from a $\mathrm{PH}(3)$ point if possible.

We start by considering cases for which $n_{2}=1.45$. Based on the results presented in Section 4.1, with this value of $n_{2}$ the interval of realizable third normalized moments is $(1.6517,2.1498)$ with $\mathrm{ME}(3)$ while it is $(1.8457,1.9573)$ 
with $\mathrm{PH}(3)$. First we look at the middle point of the $n_{3}$ interval that can be realized with a $\mathrm{PH}(3)$, i.e., $n_{3}=1.9015$. Figure 2 depicts the realizable region of $n_{4}$ and $n_{5}$ for both $\mathrm{PH}(3)$ and $\mathrm{ME}(3)$. In all the figures we have $n_{4}$ on the $\mathrm{x}$-axes and $n_{5}$ on the y-axes. Further, the lighter gray region contains the points that are realized with a $\mathrm{ME}(3)$ or $\mathrm{PH}(3)$ with one real and a pair of complex eigenvalues (class D) while the darker gray area contains points where the $\mathrm{ME}(3)$ or $\mathrm{PH}(3)$ is realized with three real eigenvalues. It is clear from Figure 2 that the $\mathrm{ME}(3)$ gives much higher flexibility than the $\mathrm{PH}(3)$ does. In Figure 3 we concentrate on the lower peak of the regions depicted in Figure 2. $\mathrm{ME}(3)$ is somewhat more flexible in this subregion as well and one can observe that the flexibility is increased both for what concerns the distribution with one real and two complex eigenvalues and for what concerns the distributions with three real eigenvalues.
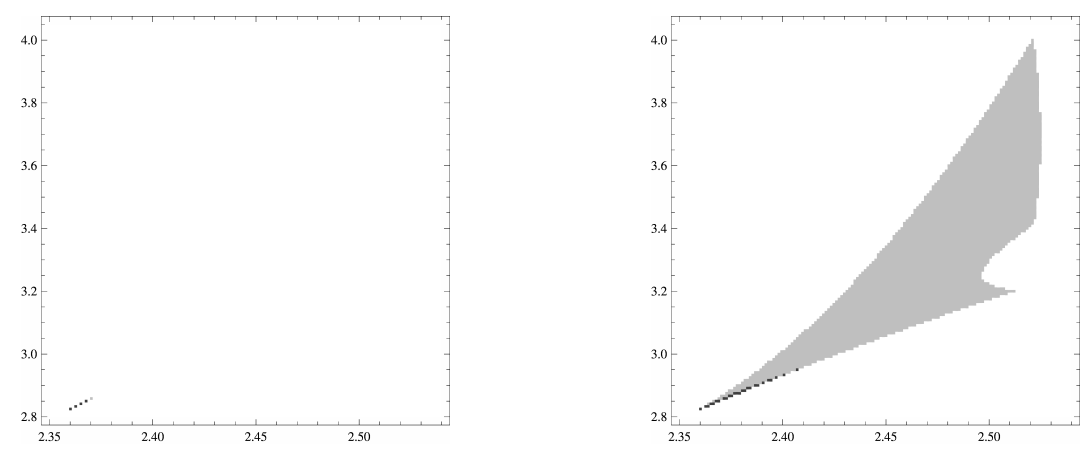

Fig. 2. Realizable $n_{4}, n_{5}$ normalized moments with $\mathrm{PH}(3)$ (on the left) and $\mathrm{ME}(3)$ (on the right) in case of $n_{2}=1.45$ and $n_{3}=1.9015$
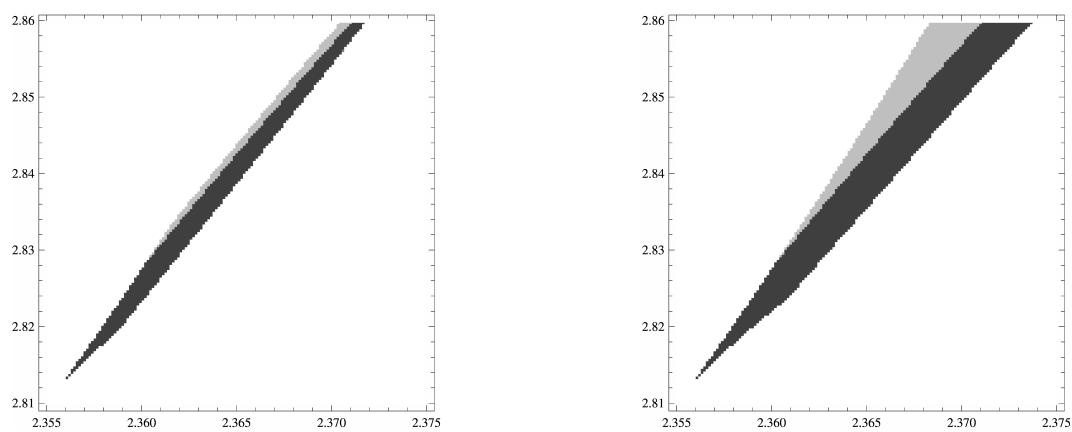

Fig. 3. Lower peak of the realizable $n_{4}, n_{5}$ region with $\mathrm{PH}(3)$ (on the left) and $\mathrm{ME}(3)$ (on the right) in case of $n_{2}=1.45$ and $n_{3}=1.9015$ 
Now we turn our attention to such $n_{3}$ values that cannot be realized by a $\mathrm{PH}(3)$ with $n_{2}=1.45$. In particular, Figure 4 depicts the realizable $n_{4}, n_{5}$ regions for $n_{2}=1.45, n_{3}=1.725$ and $n_{2}=1.45, n_{3}=2.1$ which lie respectively beneath and above the $n_{3}$ interval that can be realized with $\mathrm{PH}(3)$. By comparison with Figure 2 it is clear that approaching the possible minimum and maximum values of $n_{3}$ the realizable $n_{4}, n_{5}$ region not only changes its shape but it is shrinking as well. To illustrate further this shrinking, Figure 5 depicts the realizable $n_{4}, n_{5}$ region for $n_{2}=1.45, n_{3}=2.1249$ and $n_{2}=1.45, n_{3}=2.1373$ where the realizable region gets narrower and shorter.
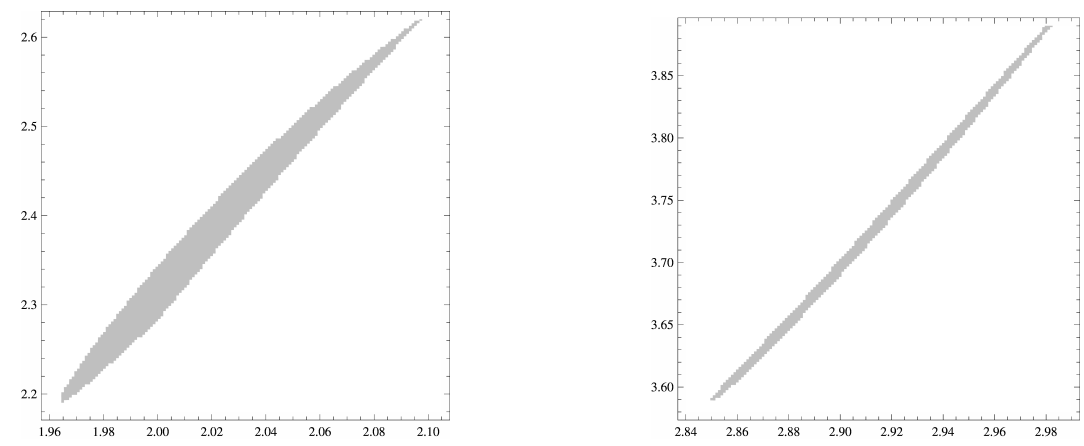

Fig. 4. Realizable $n_{4}, n_{5}$ normalized moments with $\mathrm{ME}(3)$ for $n_{2}=1.45$ and $n_{3}=1.725$ (on the left) and $n_{2}=1.45$ and $n_{3}=2.1$ (on the right)
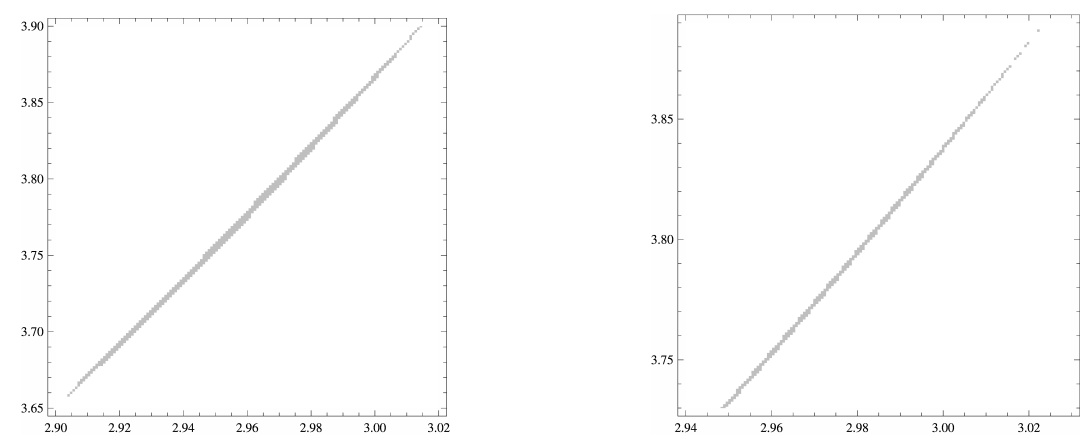

Fig. 5. Realizable $n_{4}, n_{5}$ normalized moments with $\mathrm{ME}(3)$ for $n_{2}=1.45$ and $n_{3}=$ 2.1249 (on the left) and $n_{2}=1.45$ and $n_{3}=2.1373$ (on the right)

Next we investigate a few cases with $n_{2}=1.6$. We start with two such values of $n_{3}$, namely 1.9 and 2.0, that cannot be realized with a $\mathrm{PH}(3)$. The realizable $n_{4}, n_{5}$ pairs are depicted in Figure 6 . Diverging from the minimal $n_{3}$ value, i.e. by 
increasing the actual value of $n_{3}$ the realizable region becomes larger. Diverging further from the minimal $n_{3}$ value, we choose $n_{3}=2.2$ which can be realized by $\mathrm{PH}(3)$. Figure 7 depicts the lower peak of the realizable $n_{4}, n_{5}$ region for $\mathrm{PH}(3)$ and $\mathrm{ME}(3)$. This figure reports new qualitative properties. It indicates that the realizable $n_{4}, n_{5}$ region can be composed by more than two areas and the arias are not concave. The $n_{2}=1.6, n_{3}=2.2$ case is further illustrated by Figure 8 , there is no upper bound for $n_{4}$ and $n_{5}$. Figure 9 illustrates instead how the realizable $n_{4}, n_{5}$ is changed and moved by increasing $n_{3}$ to 2.3 .
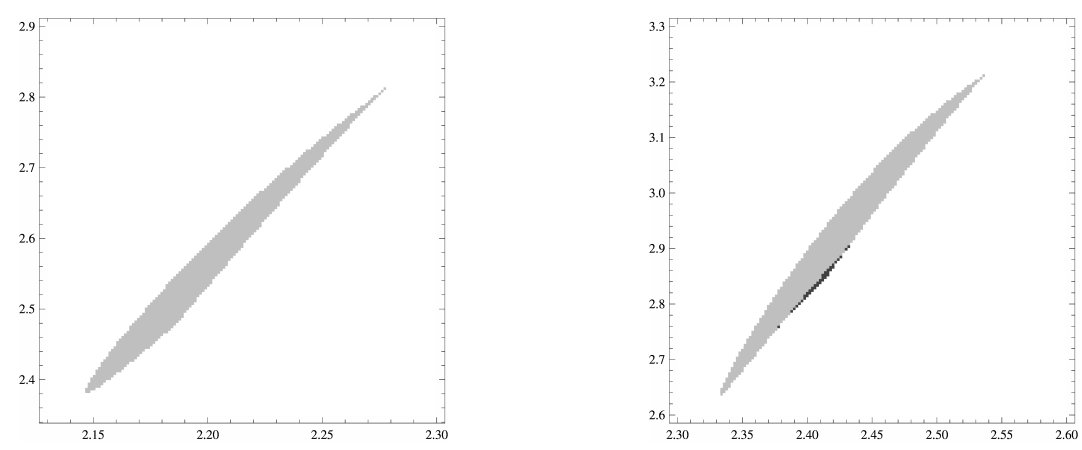

Fig. 6. Realizable $n_{4}, n_{5}$ normalized moments with $\mathrm{ME}(3)$ for $n_{2}=1.6$ and $n_{3}=1.9$ (on the left) and $n_{2}=1.6$ and $n_{3}=2.0$ (on the right)
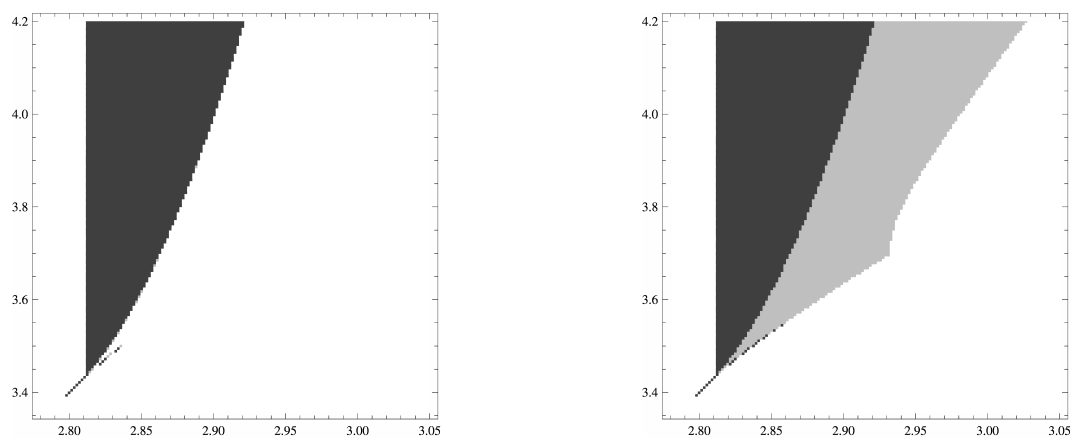

Fig. 7. Lower peak of the realizable $n_{4}, n_{5}$ region with $\mathrm{PH}(3)$ (on the left) and $\mathrm{ME}(3)$ (on the right) in case of $n_{2}=1.6$ and $n_{3}=2.2$

In the following we investigate cases with $n_{2}=2.2$. Figure 10 depicts the realizable region for $n_{3}=2.7333$ which cannot be realized by $\mathrm{PH}(3)$ and $n_{3}=$ 2.9333 which is the lower limit for $\mathrm{PH}(3)$, i.e., in this point a single $\left(n_{4}, n_{5}\right)$ point can be realized with $\mathrm{PH}(3)$. For $n_{3}=3.1333$ the regions are shown in Figure 11 

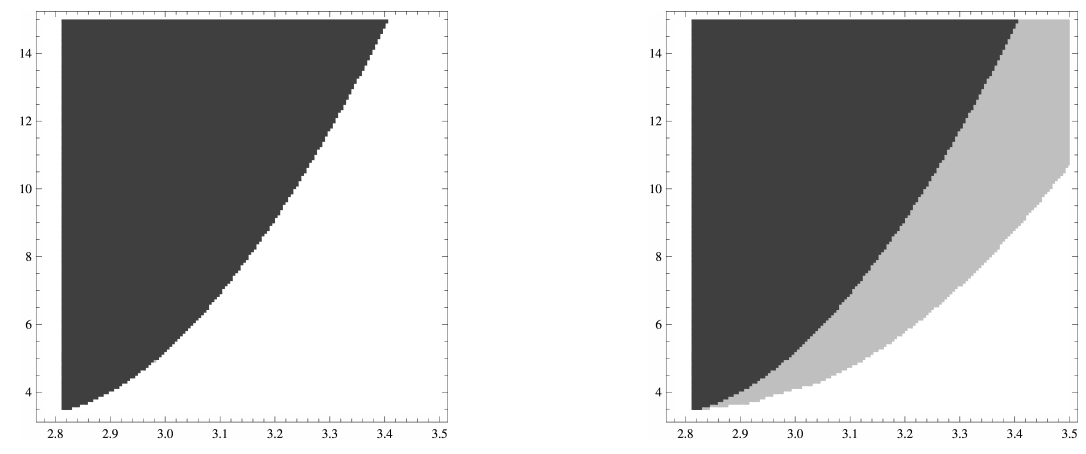

Fig. 8. Realizable $n_{4}, n_{5}$ region with $\mathrm{PH}(3)$ (on the left) and $\mathrm{ME}(3)$ (on the right) in case of $n_{2}=1.6$ and $n_{3}=2.2$
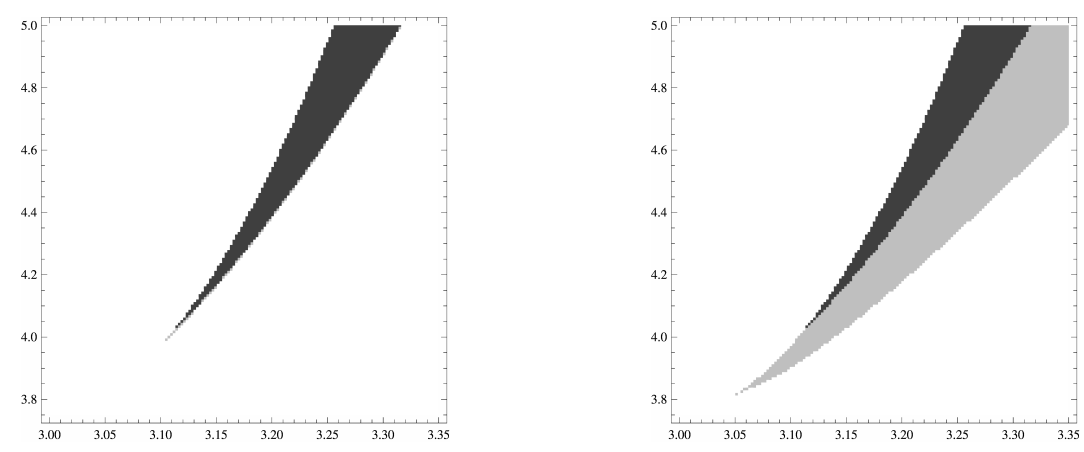

Fig. 9. Realizable $n_{4}, n_{5}$ region with $\mathrm{PH}(3)$ (on the left) and $\mathrm{ME}(3)$ (on the right) in case of $n_{2}=1.6$ and $n_{3}=2.3$ 
and for $n_{3}=3.3333$ in Figure 12. With $n_{3}=3.1333$ there are upper bounds for $n_{4}$ and $n_{5}$ which are not present with $n_{3}=3.3333$.
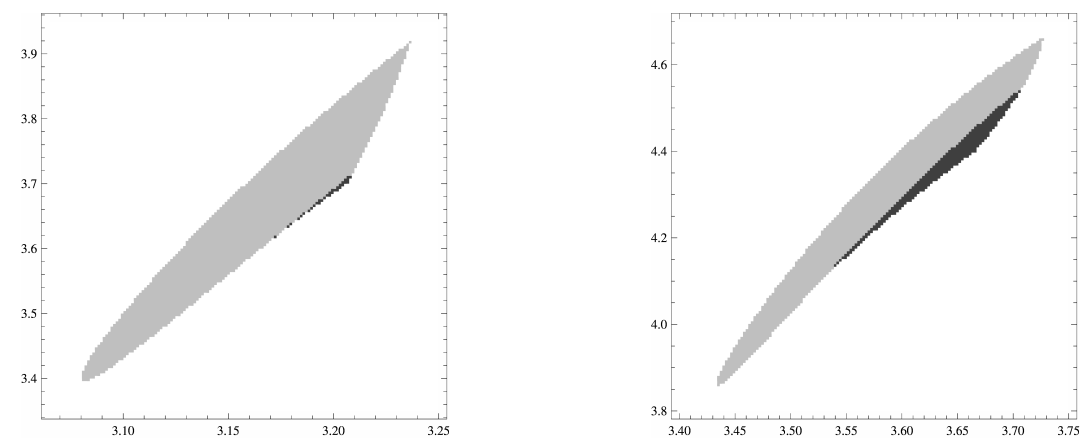

Fig. 10. Realizable $n_{4}, n_{5}$ region with $\operatorname{ME}(3)$ for $n_{3}=2.7333$ (on the left) and $n_{3}=$ 2.9333 (on the right) in case of $n_{2}=2.6$
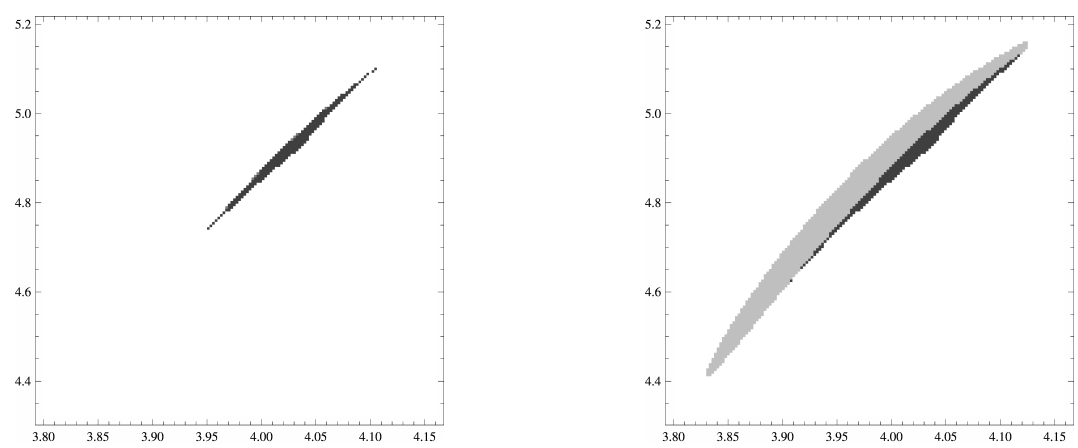

Fig. 11. Realizable $n_{4}, n_{5}$ region with $\mathrm{PH}(3)$ (on the left) and $\mathrm{ME}(3)$ (on the right) in case of $n_{2}=2.2$ and $n_{3}=3.1333$

\section{Conclusions}

This paper is devoted to the investigation of the border of ME(3) distributions. To this end we collected necessary and sufficient conditions for different kinds of order 3 matrix exponential functions to be non-negative. It turned out that these conditions are explicit in some cases, but they require the solution of a transcendent equation in other cases. Due to this fact, only numerical methods are available for the investigation of $\mathrm{ME}(3)$ borders. 

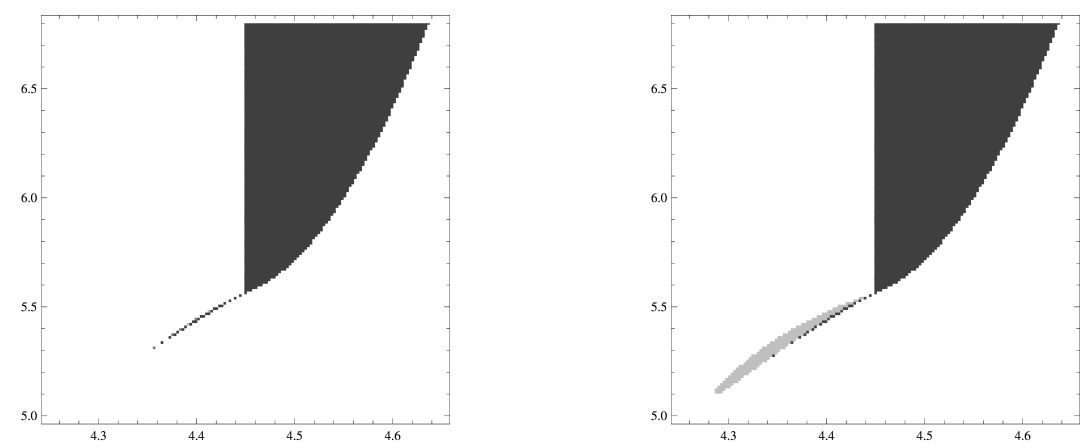

Fig. 12. Realizable $n_{4}, n_{5}$ region with $\mathrm{PH}(3)$ (on the left) and $\mathrm{ME}(3)$ (on the right) in case of $n_{2}=2.2$ and $n_{3}=3.3333$

Using those necessary and sufficient conditions we completed a set of numerical evaluations. The results show, in accordance with the common expectations, that the $\mathrm{ME}(3)$ set has very complex moments borders and it is significantly larger than the $\mathrm{PH}(3)$ set.

\section{References}

1. S. Asmussen and C. A. O'Cinneide. Matrix-exponential distributions - distributions with a rational Laplace transform. In S. Kotz and C. Read, editors, Encyclopedia of Statistical Sciences, pages 435-440, New York, 1997. John Wiley \& Sons.

2. Nigel G. Bean and Bo Friis Nielsen. Quasi-birth-and-death processes with rational arrival process components. Technical report, Informatics and Mathematical Modelling, Technical University of Denmark, DTU, IMM-Technical Report-200720, 2007.

3. A. Bobbio, A. Horváth, and M. Telek. Matching three moments with minimal acyclic phase type distributions. Stochastic models, pages 303-326, 2005.

4. T. Éltető, S. Rácz, and M. Telek. Minimal coefficient of variation of matrix exponential distributions. In 2nd Madrid Conference on Queueing Theory, Madrid, Spain, July 2006. abstract.

5. Mark William Fackrell. Characterization of matrix-exponential distributions. Technical report, School of Applied Mathematics, The University of Adelaide, Ph. D. thesis, 2003.

6. G. Horváth and M. Telek. On the canonical representation of phase type distributions. Performance Evaluation, 2008. doi:10.1016/j.peva.2008.11.002.

7. G. Latouche and V. Ramaswami. Introduction to matrix analytic methods in stochastic modeling. SIAM, 1999.

8. L. Lipsky. Queueing Theory: A linear algebraic approach. MacMillan, New York, 1992.

9. M. Neuts. Matrix-Geometric Solutions in Stochastic Models. John Hopkins University Press, Baltimore, MD, USA, 1981. 
10. J.F. Prez, J. Van Velthoven, and B. Van Houdt. Q-mam: A tool for solving infinite queues using matrix-analytic methods. In Proceedings of SMCtools'08, Athens, Greece, 2008. ACM Press.

11. M. Telek and G. Horváth. A minimal representation of Markov arrival processes and a moments matching method. Performance Evaluation, 64(9-12):1153-1168, Aug. 2007.

12. A. van de Liefvoort. The moment problem for continuous distributions. Technical report, University of Missouri, WP-CM-1990-02, Kansas City, 1990. 